\title{
Estudio de la Inteligencia Emocional y Función Ejecutiva en Educación Primaria
}

\author{
Edurne Rebollo Goñi. Centros Auditivos "GAES" de Irún (Gipuzkoa) \\ Cristina de la Peña Álvarez. Universidad Francisco de Vitoria \\ Recepción: 13 de diciembre de 2016 | Aceptado: 10 de enero de 2017 \\ Correspondencia: Cristina de la Peña Álvarez | Correo-e: cristina.delapena@ufv.es \\ (iD 0000-0003-1176-4981 \\ Citar: Rebollo, E. y de la Peña, C. (2017). Estudio de la Inteligencia Emocional y Función \\ Ejecutiva en Educación Primaria. ReiDoCrea, 6, 29-36.
}

\begin{abstract}
Resumen: La Inteligencia Emocional y la Función Ejecutiva son clave en la educación por la influencia en el proceso de enseñanza-aprendizaje. El objetivo de este trabajo es analizar la relación entre la Inteligencia Emocional (intrapersonal e interpersonal) y la Función Ejecutiva. Método: Los participantes $(\mathrm{N}=87, \mathrm{M}=7.50, \mathrm{SD}=1.03)$ eran escolares de Educación Primaria, a los que se les administró la prueba de Evaluación Neuropsicológica de Funciones Ejecutivas en niños (Portellano, Martínez y Zumárraga, 2009) para valorar las funciones ejecutivas, concretamente planificación, fluidez verbal e interferencia cognitiva y el Cuestionario de Inteligencias Múltiples de Armstrong (2001) con las escalas de inteligencia interpersonal e inteligencia intrapersonal para valorar Inteligencia Emocional. Se emplea un diseño ex postfacto, descriptivo y correlacional. Resultados: El análisis evidencia que existen relaciones significativas entre Inteligencia Emocional y Funciones Ejecutivas. Conclusiones: Resalta la relevancia de incluir en el currículo educativo programas de intervención neuropsicológica que trabajen habilidades emocionales y competencias ejecutivas para optimizar el rendimiento académico y personal del alumnado de Educación Primaria.
\end{abstract}

Palabras clave: Inteligencia Emocional | Función Ejecutiva

\section{Study of Emotional Intelligence and Executive Function in Primary Education}

\begin{abstract}
Emotional Intelligence and Executive Function are key in education due to their influence in the teaching-learning process. The aim of this research study is to analyze the relationship between emotional intelligence (intra and interpersonal) and some of the components of executive functions. Method: The participants $(\mathrm{N}=87, \mathrm{M}=7.50, \mathrm{SD}=1.03)$ are students in Primary Education who were administered the Neuropsychological Assessment of Executive Functions in Children (Portellano, Martínez y Zumárraga, 2009 ) to assess executive functions, specifically planning, verbal fluency and cognitive interference as well as Armstrong's questionnaire on Multiples Intelligences (2001) with scales of interpersonal intelligence and intrapersonal intelligence to assess emotional intelligence. An ex post facto descriptive and correlational design was used. Results: The analysis shows that there are significant relationships between emotional intelligence and executive functions. Conclusions: The relevance of including in the educational curriculum neuropsychological intervention programs that develop emotional skills and executive competencies to optimize the academic and personal performance of students of primary education is highlighted.
\end{abstract}

Key words: Emotional Intelligence | Executive Function

\section{Introducción}

Estudios actuales (Alonso, 2014; Bello, Rionda y Rodríguez, 2010) destacan el papel modelador de las emociones y su importante función en la regulación de la conducta humana y en el rendimiento educativo. Sin embargo para Gardner (2011) la Inteligencia Emocional la conforman la inteligencia intrapersonal e interpersonal. La "inteligencia intrapersonal" constituye la competencia de alcanzar los sentimientos propios y las emociones íntimas, el reflexionar sobre los procesos de pensamiento (metacognición); conlleva además la capacidad de autorreflexión y la intuición; y, la "inteligencia interpersonal" hace referencia a la capacidad de notar y conocer a otras personas, sentir empatía hacia los demás y ofrecer una respuesta acorde a sus 
inquietudes, necesidades o deseos. Actualmente, Mayer, Salovey, Caruso y Cherkasskiy (2011) definen Inteligencia Emocional como la habilidad para gestionar las emociones de cada uno y las de los demás, conocer la diferencia entre ellas y con toda la información aprovecharla para orientar el propio pensamiento y acciones.

Según Goleman (2012) la Inteligencia Emocional es una capacidad aprendida y susceptible de desarrollo mediante el entrenamiento en la percepción de uno mismo, autorregulación, automotivación, empatía y capacidad de relación y, para Bisquerra, Pérez-González y García (2015) la educación de la inteligencia emocional es una innovación educativa que se dirige al desarrollo de las capacidades emocionales para contribuir al bienestar personal y social.

Desde la neurobiología (Sánchez y Román, 2004) se aportan datos sobre la influencia de componentes ejecutivos en el control emocional de la Inteligencia emocional. Esta idea está en consonancia con la teoría de la complejidad de Zelazo, Carter, Reznick y Frye (1997) que plantean la interacción de aspectos emocionales, cognitivos y ejecutivos en la inteligencia emocional.

La Función Ejecutiva es un concepto que posee gran complejidad ya que engloba a un conjunto amplio de funciones de autorregulación permitiendo controlar, organizar y coordinar otras funciones cognitivas, respuestas emocionales y comportamientos con el fin de alcanzar una meta propuesta además de inhibir información irrelevante (Castillo, Flores, Jiménez, 2014). Actualmente, los autores (Fuster, 2014; Tirapu, García, Ríos y Ardila, 2011) coinciden en considerar la Función Ejecutiva como una agrupación de capacidades cognitivas que controlan los procesos cognitivos y que incluyen aspectos del comportamiento humano como la definición de objetivos, decidir o seleccionar pensamientos y acciones. Entre los procesos cognitivos enmarcados dentro del marco de las Funciones Ejecutivas se encuentran la abstracción (capacidad de analizar la información implícita presentada), atención sostenida: (habilidad para mantener fija la atención en una situación concreta por un tiempo considerable), autorregulación (capacidad para controlar y gestionar adecuadamente las propias emociones), control metacognitivo (regulación en los procesos cognitivos), flexibilidad mental (capacidad para cambiar un patrón de respuestas y tareas y adaptarse a nuevas circunstancias), fluidez (capacidad de generar un tipo de información que la situación exige en un momento determinado), inhibición (capacidad para suprimir una respuesta dominante), y memoria de trabajo (capacidad para mantener cierta información por un tiempo corto mientras se realiza una acción basada en esta información).

Las investigaciones sobre neuroimagen (Arteaga y Quebradas, 2010; Stuss y Alexander, 2007) ponen de manifiesto que la función ejecutiva depende de la actividad cerebral del lóbulo prefrontal, de las estructuras subcorticales y de los circuitos córticosubcorticales. De esta manera, la Función Ejecutiva está en continua relación con la inteligencia emocional dado el carácter de control sobre todos los procesos, ayudando al control y manejo de las emociones, que son importantes para tener un buen rendimiento escolar y necesarias para superar con éxito las demandas del aprendizaje (Stelzer y Cervigni, 2011). En la revisión de la literatura científica, diversos estudios (Cabello, Ruiz y Fernández, 2010; Mestre, Guil, Lopes, Salovey y Gil-Olarte, 2006) indican que las tareas escolares y su correspondiente crecimiento intelectual conllevan la competencia de utilizar y organizar las emociones, incrementar la concentración, controlar la conducta impulsiva y saber controlarse estando sometido a situaciones estresantes; por lo que, las competencias de tipo emocional pueden ayudar a la adecuación en el ámbito social y académico. 
El objetivo de este trabajo es analizar la relación entre Inteligencia Emocional y Función Ejecutiva en alumnado de educación primaria y tiene como hipótesis que existe relación significativa entre la Intelgiencia Emocional y la Función Ejecutiva.

\section{Método}

\section{Participantes}

En esta investigación participaron 87 escolares de ambos sexos que cursaban $1^{\circ}, 2^{\circ}$ y $3^{\circ}$ de Educación Primaria de un colegio concertado de Irún (Gipuzkoa). La distribución de la muestra en cuanto al curso y sexo se muestra en la tabla 1, observando un mayor porcentaje de niñas $(56.32 \%)$ frente a niños $(43.68 \%)$ y mayor número de niños en segundo curso $(36.78 \%)$, seguido de tercer curso $(33.33 \%)$ y primer curso $(29.88 \%)$.

\begin{tabular}{|lcccc|}
\hline \multicolumn{5}{|c|}{ Tabla 1. Distribución de la muestra según género y curso educativo } \\
\hline \multirow{3}{*}{ Niños } & $1^{\circ}$ curso & $2^{\circ}$ curso & $3^{\circ}$ curso & Total \\
\cline { 2 - 5 } & 10 & 13 & 15 & 38 \\
Niñas & $11,49 \%$ & $14,94 \%$ & $17,24 \%$ & $43,68 \%$ \\
\cline { 2 - 5 } & 16 & 19 & 14 & 49 \\
Total & $18,39 \%$ & $21,84 \%$ & $16,09 \%$ & $56,32 \%$ \\
\cline { 2 - 5 } & 26 & 32 & 29 & 87 \\
\hline
\end{tabular}

El rango de edad del alumnado de la muestra fue de 6 a 9 años ( $M=7.50 ; D T=1.03)$, constituyendo el $19 \%$ los niños de 6 años, el $31 \%$ los de 7 años, el $29 \%$ los de 8 años y el $21 \%$ los de 9 años.

Los criterios de inclusión de la muestra fueron: pertenecer a los cursos de $1^{\circ}, 2^{\circ}$ y $3^{\circ}$ de educación primaria, asistir de forma regular a las clases, no presentar ningún trastorno neurológico y/o psicopatológico grave y tener el consentimiento firmado de padres o tutores para la administración de las pruebas.

\section{Instrumentos}

Los instrumentos utilizados en esta investigación son dos pruebas. La primera prueba es la Evaluación Neuropsicológica de las Funciones Ejecutivas en Niños ENFEN (Portellano, Martínez y Zumárraga, 2009). Este test permite evaluar las Funciones Ejecutivas en niños entre 6 y 12 años de edad mediante diferentes pruebas. En este trabajo se han utilizado concretamente las tareas de fluidez verbal, anillas y colores. En la tarea de fluidez verbal, el alumno nombra el número máximo de palabras diferentes que comienzan por una letra determinada en un minuto y se anotan los errores cometidos. En la prueba anillas, al alumnado se le da un tablero con tres ejes verticales, anillas de colores y una lámina modelo; tiene que ir colocando las anillas de manera creciente entre tres y seis colores diferentes en la misma posición que presenta la lámina modelo. Para el punto de partida el instructor le pone las anillas, en un orden determinado, en el eje izquierdo y el alumno debe realizar movimientos hasta conseguir la torre modelo en el eje derecho. Así, ha de obtener el modelo de la lámina en el menor tiempo posible, con el menor número de movimientos y con una dificultad creciente. Se cronometra el tiempo empleado en la realización de la prueba y se anotan los errores cometidos en el sentido de colocación de las fichas en otro orden de colores diferente al modelo aunque luego el alumno rectifique su error. En la prueba de interferencias, se presenta al alumno una lista de palabras con nombres de diferentes colores pero impresas en tintas diferentes del color escrito (ej. La palabra AZUL escrita en color rojo); el alumno debe decir el color en que está escrita la palabra 
y debe realizarlo en el menor tiempo posible y con el menor número de errores. Se anotan los errores cometidos en los casos en que no coincida el nombre del color de la palabra con lo mencionado por el alumno. Además se cronometra el tiempo empleado en la realización de la tarea.

La segunda prueba es el Cuestionario de inteligencias múltiples de Armstrong (2001). Consiste en una serie de cuestionarios que permiten valorar las distintas inteligencias múltiples. En este trabajo, se utilizan los cuestionarios de inteligencia intrapersonal y de inteligencia interpersonal, que conforman y aportan una medida de la Inteligencia Emocional. En ambos cuestionarios, los tutores tienen que rellenar los ítems según la información que los niños han ido proporcionándoles a lo largo del curso. En cuanto a la inteligencia intrapersonal, se trata de valorar diez ítems relacionados con el pensamiento propio, autonomía, planificación, organización de ideas...o la conciencia de las propias emociones y la capacidad para expresar sentimientos. Por lo que respecta a la inteligencia interpersonal también se detallan diez ítems relacionados con la manera en la que los niños dirigen, organizan, se relacionan y cómo hacen frente a las situaciones que se les presentan en su vida diaria. Los dos cuestionarios se valoran de la siguiente manera: "sí" equivale a 1 punto, "no" a 0 puntos y "al" que significa, algunas veces: 0,5 puntos. Una vez resueltos los ítems con sus correspondientes puntuaciones se hace la suma total, de 0 a 10 , por lo que cada cuestionario de inteligencia tendrá una puntuación global. El coeficiente de fiabilidad de consistencia interna (a de Cronbach) para el cuestionario de inteligencia interpersonal es 0.78 y para el cuestionario de inteligencia intrapersonal es 0.69 (Alaga et al., 2012).

\section{Diseño}

Se trata de un diseño no experimental ya que no existe ningún control sobre las variables, no se lleva a cabo ningún tratamiento o intervención y no se ha procedido a una selección y asignación de la muestra de manera aleatoria, sino que componen una muestra incidental. Esta investigación cumple con los requisitos de un diseño ex post facto, en el que se recogen y analizan datos de fenómenos ya acontecidos. Es descriptivo y correlacional porque describe los valores de las variables y analiza el tipo de relación entre ellas.

\section{Procedimiento}

Se llevó a cabo una entrevista con el director del centro educativo participante y con las tutoras de los cursos en los que se pretende llevar a cabo el trabajo para exponer los objetivos de la investigación, describir las pruebas a aplicar a los alumnos y una vez explicado, solicitar su permiso y promover su colaboración. Posteriormente, se solicitó el consentimiento informado por escrito de los padres autorizando a sus hijos a participar en la investigación.

El espacio donde se llevaron a cabo las pruebas fue el aula contigua a la sala de profesores. Para que no existieran variables extrañas, todos los alumnos siguieron el mismo orden de realización de las pruebas: en primer lugar con la prueba de Fluidez verbal, seguidamente la de Planificación y por último la prueba de Interferencias. El tiempo de aplicación aproximado fue de 20 minutos para la pruebas ENFEN (Portellano et al, 2009). El investigador estuvo presente durante la aplicación de las pruebas para aclarar posibles dudas de los alumnos en lo que respecta a su realización. 
Las tutoras de los cursos implicados rellenaron los cuestionarios de Inteligencia Emocional de cada uno de sus alumnos teniendo en cuenta el comportamiento de los niños en el aula. El orden de cumplimentación de los mismos fue primeramente el cuestionario sobre Inteligencia Intrapersonal para continuar con el cuestionario de Inteligencia Interpersonal. El tiempo necesario para su cumplimentación fue de unos 10 minutos por cada alumno.

\section{Análisis de Datos}

Tras la recogida de datos se ha procedido a realizar análisis descriptivos de la muestra y análisis correlacionales entre las distintas variables a través del Coeficiente de Correlación de Pearson con un nivel de significación de $\alpha=.05$. Para el análisis de datos, se ha utilizado el paquete estadístico SPSS versión 19.0 para Windows.

\section{Resultados}

En la tabla 2, se muestran los resultados de los estadísticos descriptivos de la variable Inteligencia Emocional. A nivel general, se observa que las medias de inteligencia interpersonal e intrapersonal son similares, siendo ligeramente superiores en Inteligencia Interpersonal. Por sexos, se observa que las niñas tienen más media en Inteligencia Interpersonal que los niños y en Inteligencia Intrapersonal las medias son muy similares.

\begin{tabular}{|lcccccr|}
\hline \multicolumn{7}{|c|}{ Tabla 2. Estadísticos descriptivos de Inteligencia Emocional } \\
\hline & \multicolumn{5}{c|}{ I.Interpersonal } & \multicolumn{3}{c|}{ I. Intrapersonal } \\
\cline { 2 - 7 } Media & General & Niños & Niñas & General & Niños & Niñas \\
\cline { 2 - 7 } Desviación Típica & 6.92 & 6.68 & 7.11 & 6.75 & 6.75 & 6.78 \\
& 1.22 & 1.28 & 1.16 & 1.17 & 1.31 & 1.06 \\
\hline
\end{tabular}

En cuanto a los resultados obtenidos por cursos educativos, en inteligencia intrapersonal el tercer curso $(M=7.086)$ tiene más media seguido de primero $(M=6.635)$ y segundo curso $(M=6.547)$. En inteligencia interpersonal, la mayor media la posee el tercer curso $(M=7.210)$ seguido de segundo curso $(M=6.922)$ y primer curso $(M=$ $6.615)$.

En la tabla 3, se muestran los resultados estadísticos de la variable Función Ejecutiva; observando a nivel general, que la media de los errores cometidos en fluidez verbal e interferencia cognitiva son similares siendo ligeramente inferior en la tarea de planificación. En lo que respecta al tiempo medido, la media se observa mayor en la tarea de planificación que en la de interferencia cognitiva. Respecto al sexo, los niños cometen más errores que las niñas en todas las tareas ejecutivas valoradas; concretamente los chicos se equivocan más en tareas de interferencia cognitiva, seguido de actividades en fluidez verbal y planificación y las chicas cometen más errores primero en tareas de fluidez verbal, seguido de ejercicios de interferencia cognitiva y planificación. Además, las niñas realizan las tareas requiriendo ligeramente un menor tiempo que los niños tanto en ejercicios de planificación como de interferencia cognitiva.

\begin{tabular}{|llllll|}
\hline \multicolumn{5}{c}{ Tabla 3. Estadísticos descriptivos de Función ejecutiva a nivel general } \\
\hline & Fluidez Verbal & Planificación & Interferencia & Planificación & Interferencia \\
& errores & errores & Cognitiva errores & tiempo & Cognitiva tiempo \\
\cline { 2 - 6 } Media Total & 2,207 & 1,701 & 2,287 & 294,310 & 261,931 \\
Desviación típica & 1,221 & 1,142 & 1,372 & 24,771 & 22,105 \\
Media Niños & 2,34 & 1,86 & 2.76 & 298,26 & 267,26 \\
Media Niñas & 2,10 & 1,57 & 1,91 & 291,24 & 257,79 \\
\hline
\end{tabular}


Por cursos educativos, el primer curso es el que presenta la mayor media en errores tanto en fluidez, planificación e interferencia cognitiva, seguido del segundo curso y del tercer curso. En primer curso, se cometen más errores en interferencia cognitiva, seguido de fluidez verbal y planificación; en segundo curso se cometen más errores en fluidez verbal seguido de interferencia cognitiva y planificación; y, en tercer curso se cometen más errores en interferencia cognitiva, seguido de fluidez verbal y planificación. A nivel general, destaca el mayor número de errores en la tarea de interferencia cognitiva siendo mayor que en las tareas de fluidez verbal y planificación. Es en la tarea de planificación donde se produce un descenso más acusado a medida que avanza la edad.

Los resultados del análisis correlacional, mediante Correlación de Pearson, confirman que sí existe relación significativa entre la Inteligencia Emocional y las Funciones Ejecutivas evaluadas en este estudio; concretamente, se trata de una relación significativa negativa porque cuando se produce un aumento en la variable Inteligencia Emocional (intrapersonal e interpersonal) se produce directamente un descenso del número de errores cometidos y menor el tiempo empleado en las diferentes tareas de las funciones ejecutivas analizadas. La relación entre los errores en fluidez verbal y la inteligencia intrapersonal $(p=-.736 ; p=.000)$ es una correlación significativa negativa alta, es decir, que un aumento alto en inteligencia intrapersonal produce directamente un descenso alto del número de errores en tareas de fluidez verbal y viceversa. La correlación entre Inteligencia Interpersonal y los errores en fluidez verbal $(r=-.708$; $p=.000$ ) es significativa negativa alta, es decir, que un aumento alto en inteligencia interpersonal produce directamente un descenso alto del número de errores en tareas de fluidez verbal y viceversa. La correlación entre los errores en planificación y la inteligencia intrapersonal $(r=-.727 ; p=.000)$ es significativa negativa alta, es decir, que un aumento alto en inteligencia intrapersonal produce directamente una disminución alta del número de errores en tareas de planificación y viceversa. La correlación entre los errores en planificación y la inteligencia interpersonal $(r=-.589 ; p=.000)$ es significativa negativa moderada, es decir, que un aumento moderado en inteligencia interpersonal produce directamente una disminución moderada del número de errores en tareas de planificación y viceversa. La correlación entre interferencia cognitiva y la inteligencia intrapersonal $(r=-.563 ; p=.000)$ es significativa negativa moderada, es decir, que un aumento moderado en inteligencia intrapersonal produce directamente una disminución moderada del número de errores en tareas de interferencia cognitiva y viceversa. La correlación entre interferencia cognitiva errores y la inteligencia interpersonal $(r=-.495 ; p=.000)$ es significativa negativa moderada, es decir, que un aumento moderado en inteligencia interpersonal produce disminución moderada del número de errores en tareas de interferencia cognitiva y viceversa. La correlación entre planificación tiempo y la inteligencia intrapersonal $(r=-.647 ; p=.000)$ es significativa negativa moderada, es decir, que un aumento moderado en inteligencia intrapersonal produce directamente una disminución moderada del tiempo que se tarda en realizar tareas de planificación y viceversa. La correlación entre planificación tiempo y la inteligencia interpersonal $(r=-.652 ; p=.000)$ es significativa negativa moderada, es decir, que un aumento moderado en inteligencia interpersonal produce directamente un descenso moderado del tiempo que se tarda en realizar tareas de planificación y viceversa. La correlación entre interferencia cognitiva tiempo y la inteligencia intrapersonal $(r=-.561 ; p=.000)$ es significativa negativa moderada, es decir, que un aumento moderado en inteligencia intrapersonal produce directamente una disminución moderada del tiempo que se tarda en realizar tareas de interferencia cognitiva y viceversa. La correlación entre interferencia cognitiva tiempo y la inteligencia interpersonal $(r=-.591 ; p=.000)$ es significativa negativa moderada, es decir, que un aumento moderado en inteligencia interpersonal produce directamente un descenso moderado del tiempo que se tarda en realizar tareas de interferencia cognitiva y viceversa. 


\section{Discusión}

Esta investigación verifica la relación existente entre la Inteligencia Emocional y las Funciones Ejecutivas. De tal forma que, trabajando la Inteligencia Emocional se mejoran las Funciones Ejecutivas porque se disminuyen el número de errores y el tiempo empleado en las tareas ejecutivas. Existen estudios (Cabello et al, 2010; Mestre et al., 2006) que indican los beneficios de una gestión correcta de las habilidades emocionales en el proceso de aprendizaje de los alumnos mejorando la adaptación social y académica.

En cuanto a la Inteligencia Emocional, la inteligencia interpersonal presenta valores ligeramente superiores a la inteligencia intrapersonal. Desde la perspectiva del género, las chicas tienen más media que los chicos tanto en inteligencia interpersonal como en inteligencia intrapersonal (aunque en esta última es ligeramente superior en tres centésimas). Estos datos están en la misma línea que los encontrados por Ciarrohi, Chan y Bajgar (2001) en su estudio con adolescentes, en el que las chicas tuvieron más media en inteligencia emocional que los chicos. En función de los cursos educativos, se observa cómo la inteligencia emocional aumenta conforme avanza la edad de acuerdo al desarrollo cognitivo, presentando el curso de tercero las mayores puntuaciones en inteligencia interpersonal e inteligencia intrapersonal.

En relación a la Función Ejecutiva, generalmente hay un mejor desempeño de los componentes de planificación, seguido de fluidez verbal e interferencia cognitiva puesto que se producen menos errores en tareas de planificación, seguido de fluidez verbal e interferencia cognitiva en la muestra analizada. Sin embargo, se tarda más tiempo en las tareas de planificación que en las tareas de interferencia cognitiva, lo que implica que los alumnos en las actividades de planificación tardan más tiempo pero cometen menos errores. Desde el análisis del género, tanto los chicos como las chicas realizan mejor la tarea de planificación cometiendo menos errores de todas las funciones ejecutivas estudiadas pero también es en planificación el ejercicio que tardan más tiempo en realizar. Según el curso educativo, se cometen menos errores a medida que avanza el curso y aumenta la edad, puesto que los niños de primer curso son los que tienen más errores en las actividades de las funciones ejecutivas evaluadas; este resultado es consecuente con el desarrollo evolutivo de los procesos cognitivos superiores. Huizinga, Dolan y Van der Molen (2006) encontraron una mejora de las habilidades ejecutivas desde la etapa de educación infantil hacia primaria.

Por último, en cuanto a la existencia de relación significativa negativa entre inteligencia emocional y función ejecutiva, se halla tanto en los errores como en el tiempo empleado en realizar cada tarea ejecutiva. Estos datos están en la misma dirección que los hallados por Ramos, Fernández-Berrocal y Extremera (2003) que hallan relaciones negativas entre la inteligencia emocional y aspectos ejecutivos como la inhibicción. Estas correlaciones significativas ponen de manifiesto la vinculación directa entre inteligencia emocional y funciòn ejecutiva modulándose mutuamente e influyendo en el proceso de aprendizaje y rendimiento académico del alumnado. Esta relación entre las variables estudiadas está avalada por los datos neurobiológicos (Purves, 2010) que indican la participación de la corteza prefrontal en inteligencia emocional y función ejecutiva.

En el ámbito educativo, esta relación significativa permitirá a los profesores y orientadores elaborar y aplicar programas de intervención en el aula gestionados desde el plan de acción tutorial, ya que están directamente relacionados con las líneas de acción tutorial: enseñar a pensar, enseñar a convivir, enseñar a ser persona y enseñar a tomar decisiones. El entrenamiento mediante actividades en inteligencia 
emocional facilitará la mejora de los componentes de planificación, fluidez verbal e interferencia cognitiva de la función ejecutiva lo que conllevará la optimización de la toma de decisiones de cualquier naturaleza; y, a su vez, el entrenamiento en función ejecutiva aumentará la capacidad de comprender y controlar los sentimientos propios y de otras personas, es decir, la inteligencia emocional. Estos programas de intervención en Educación Primaria han de tener una duración mínima de tres meses constantes durante todas las semanas para permitir valorar los efectos del entrenamiento, acompañados de distintas actividades que trabajen los conceptos de inteligencia emocional y función ejecutiva.

En síntesis, los resultados ofrecidos por este trabajo proporcionan nuevas líneas de investigación que permiten optimizar el rendimiento académico del alumnado, advirtiendo la relación significativa entre la inteligencia emocional y las funciones ejecutivas. Se requieren estudios de investigación más amplios que confirmen estos hallazgos y el desarrollo en los centros educativos de programas de educación emocional y de función ejecutiva que optimicen el desarrollo integral del alumnado de Educación Primaria.

Referencias

Alaga, J., Ponce, C., Blunes, M., Elizalde, R., Montgomery, W., y Gutiérrez, V. (2012). Las inteligencias múltiples: evaluación y relación con el rendimieneto en matemática en estudiantes del quinto año de secundaria de lima metropolitana. Revista de Investigación en Psicología, 15(2), 163-202.

Alonso, L. (2014). Inteligencia emocional y rendimiento académico: análisis de variables mediadoras. Trabajo Fin de Grado de Psicología, Universidad de Salamanca.

Armstrong, T. (2001) Inteligencias múltiples: cómo descubrirlas y estimularlas en sus hijos. Bogotá: Editorial Norma.

Arteaga, G. y Quebradas, D. (2010) Funciones ejecutivas y marcadores somáticos: apuestas, razón y emociones. El Hombre y la Máquina, 34, 115-129.

Bello, Z., Rionda, H. y Rodríguez, M. (2010) La inteligencia emocional y su educación. Varona, 51, 36-43.

Bisquerra, R., Pérez-González, J. y García, E. (2015). Inteligencia Emocional en la Educación. Madrid: Síntesis.

Cabello, R., Ruiz, D. y Fernández, P. (2010) Docentes emocionalmente inteligentes. REIFP, 13(1), 41-49.

Castillo, R., Flores J., \& Jiménez, N. (2014) Desarrollo de funciones ejecutivas, de la niñez a la juventud. Anales de Psicología, 30(2), 463-473.

Ciarrochi, J., Chan, A., \& Bajgar, J. (2001). Measuring emotional intelligence in adolescents. Personality and Individual Differences, 31(7), 1105-1119.

Fuster, J. (2014). Cerebro y libertad: Ios cimientos cerebrales de nuestra capacidad para elegir. Barcelona: Ariel.

Gardner, H. (2011) Inteligencias múltiples. La teoría en la práctica. Barcelona: Paidós.

Goleman, D. (2012). El cerebro y Inteligencia Emocional: nuevos descubrimientos. Barcelona: Ediciones $\mathrm{B}$.
Huizinga, M., Dolan, C., \& Van der Molen, MW. (2006) Age-related change in executive function: Developmental trends and a latent variables analysis. Neuropsychología, 44, 2017-203.

Mayer, J., Salovey, P., Caruso, D., \& Cherkasskiy, L. (2011). Emotional intelligence. En J. Stenberg, y B. Kaufman (Eds.), The CambrigeHandbook of Intelligence (528-549). Cambrige: University Press.

Mestre, J., Guil, R., Lopes, P., Salovey, P., y Gil-Olarte, P. (2006) Emotional intelligence and social and academic adaptation to school. Psicothema, 18, 112-117.

Portellano, JA., Martínez, R. y Zumárraga, L. (2009) Manual de evaluación neuropsicológica de las funciones ejecutivas en niños. Madrid: ENFEN.

Purves, D. (2010). Neurociencia. Madrid: Médica Panamericana.

Ramos, N., Fernández-Berrocal, P., \& Extremera, N. (2007). Perceived emotional intelligence facilitates cgnitive-emotional processes of adaptation to an acute stressor. Cognition and Emotion, 21(4), 758-772.

Sánchez, J. y Román, F. (2004) Amígdala, corteza prefrontal y especialización hemisférica en la experiencia y expresión emocional. Anales de psicología, 20, 223-240.

Stelzer, F. y Cervigni, M. (2011). Desempeño académico y funciones ejecutivas en infancia y adolescencia. Una revisión de la literatura. RIE, 9(1), 148-156.

Stuss, D., \& Alexander, M. (2007) Is there a dysexecutive syndrome? Philosophical Transactions of the Royal Society of London B, Biological Sciences, 362, 901-915.

Tirapu, J., García, A., Ríos, M. y Ardila, A. (2011). Neuropsicología del córtex prefrontal y de las funciones ejecutivas. Barcelona: Viguera.

Zelazo, P., Carter, A., Reznick, J., \& Frye D. (1997). Early development of executive function: a problem-solving framework. Review of General Psychology, 1, 198-226. 